\title{
The fixed point theorems of 1-set-contractive operators in Banach space
}

\author{
Shuang Wang
}

\section{Correspondence:}

wangshuang19841119@163.com School of Mathematical Sciences, Yancheng Teachers University, Yancheng, 224051, Jiangsu, PR China

\begin{abstract}
In this paper, we obtain some new fixed point theorems and existence theorems of solutions for the equation $A x=\mu x$ using properties of strictly convex (concave) function and theories of topological degree. Our results and methods are different from the corresponding ones announced by many others.
\end{abstract}

MSC: 47H09, 47H10

Keywords: 1-Set-contractive operator, Topological degree, Convex function, Concave function, Fixed point theorems

\section{Introduction}

For convenience, we first recall the topological degree of 1 -set-contractive fields due to Petryshyn [1].

Let $E$ be a real Banach space, $p \in E, \Omega$ be a bounded open subset of $E$. Suppose that $A: \bar{\Omega} \rightarrow E$ is a 1 -set-contractive operator such that

$$
\|(I-A) x-p\| \geq \delta>0, \quad \forall x \in \partial \Omega
$$

In addition, if there exists a $k$-set-contractive operator $(k<1) W: \bar{D} \rightarrow E$ such that

$$
\|A x-W x\| \leq \frac{\delta}{3}, \quad \forall x \in \partial D,
$$

then $(I-W) x \neq p, \forall x \in \partial D$, and so it is easy to see that $\operatorname{deg}(I-W, D, p)$ is well defined and independent of $W$. Therefore, we are led to define the topological degree as follows:

$$
\operatorname{deg}(I-A, D, p)=\operatorname{deg}(I-W, D, p)
$$

Without loss of generality, we set $p=\theta$ in the above definition.

Let $A: \bar{\Omega} \rightarrow E$ be a 1 -set-contractive operator. $A$ is said to be a semi-closed 1 -setcontractive operator, if $I-A$ is closed operator (see [2]).

It should be noted that this class of operators, as special cases, includes completely continuous operators, strict set-contractive operators, condensing operators, semi-compact 1-set-contractive operators and others (see [2]).

Petryshyn [1] and Nussbaum [3] first introduced the topological degree of 1-set-contractive fields, studied its basic properties and obtained fixed point theorems of 1-setcontractive operators. Amann [4] and Nussbaum [5] have introduced the fixed point

2011 Wang; licensee Springer. This is an Open Access article distributed under the terms of the Creative Commons Attribution License (http://creativecommons.org/licenses/by/2.0), which permits unrestricted use, distribution, and reproduction in any medium, provided the original work is properly cited. 
indices of $k$-set contractive operators $(0 \leq k<1)$ and condensing operators to derive some fixed point theorems. As a complement, Li [2] has defined the fixed point index of 1-setcontractive operators and obtained some fixed point theorems of 1-set-contractive operators. Recently, Li [6] obtained some fixed point theorems for 1-set-contractive operators and existence theorems of solutions for the equation $A x=\mu x$. Very recently, $\mathrm{Xu}$ [7] extended the results of $\mathrm{Li}[6]$ and obtained some fixed point theorems. In this paper, we continue to investigate boundary conditions, under which the topological degree of 1-set contractive fields, $\operatorname{deg}(I-A, \Omega, p)$, is equal to unity or zero. Consequently, we obtain some new fixed point theorems and existence theorems of solutions for the equation $A x=\mu x$ using properties of strictly convex (concave) functions. Our results and methods are different from the corresponding ones announced by many others (e.g., Li [6], Xu [7]).

We need the following concepts and lemmas for the proof of our main results.

Suppose that $A: \bar{\Omega} \rightarrow E$ is a semi-closed 1 -set-contractive operator and $\theta \notin(I-$ $A) \partial \Omega$, then, by the standard method, we can easily see that the topological degree has the basic properties as follows:

(a) (Normalization) $\operatorname{deg}(I, \Omega, p)=1$, when $p \in \Omega$; $\operatorname{deg}(I, \Omega, p)=0$, when $p \notin \Omega$;

(b) (Solution property) If $\operatorname{deg}(I-A, \Omega, \theta) \neq 0$, then $A$ has at least one fixed point in $\Omega$.

(c) (Additivity) For every pair of disjoint open subsets $\Omega_{1}, \Omega_{2}$ of $\Omega$ such that $\{x \in$ $\Omega \mid(I-A) x=0\} \subset \Omega_{1} \cup \Omega_{2}$, we have

$$
\operatorname{deg}(I-A, \Omega, \theta)=\operatorname{deg}\left(I-A, \Omega_{1}, \theta\right)+\operatorname{deg}\left(I-A, \Omega_{2}, \theta\right) .
$$

(d) (Homotopy invariance) Let $H(t, x)=H_{t}(x):[0,1] \times \bar{\Omega} \rightarrow E$ be a continuous operator such that

$$
\left\|x-H_{t}(x)\right\| \geq \delta>0, \quad \text { for }(t, x) \in[0,1] \times \partial \Omega
$$

and the measure of non-compactness $\gamma(H([0,1] \times Q)) \leq \gamma(Q)$ for every $Q \subset \bar{\Omega}$. Then $\operatorname{deg}\left(I-H_{t}, \Omega, \theta\right)=$ const, for any $t \in[0,1]$.

(e) Let $B$ be an open ball with center $\theta, A: \bar{B} \rightarrow E$ a semi-closed 1-set-contractive operator and $(I-A) x \neq 0$ for all $x \in \partial B$. Suppose that $A$ is odd on $\partial B$ (i.e., $A(-x)=$ $A x$, for $x \in \partial B)$, then $\operatorname{deg}(I-A, B, \theta) \neq 0$.

(f) (Change of base) Let $p \neq \theta$, then $\operatorname{deg}(I-A, \Omega, p)=\operatorname{deg}(I-A-p, \Omega, \theta)$.

Lemma 1.1. [7]. Let $E$ be a real Banach space, $\Omega$ a bounded open subset of $E$ and $\theta$ $\in \Omega . A: \bar{\Omega} \rightarrow$ Eis a semi-closed 1-set-contractive operator and satisfies the LeraySchauder boundary condition

$$
A x \neq t x, \quad \text { for all } x \in \partial \Omega, \quad \text { and } t \geq 1, \quad(\mathrm{~L}-\mathrm{S})
$$

then $\operatorname{deg}(I-A, \Omega, \theta)=1$ and so $A$ has a fixed point in $\Omega$.

Definition 1.2. Let $D$ be a nonempty subset of $R$. If $\phi: D \rightarrow R$ is a real function such that

$$
\varphi[t x+(1-t) y]<t \varphi(x)+(1-t) \varphi(y), \quad \forall x, y \in D, x \neq y, t \in(0,1),
$$


then $\phi$ is called strictly convex function on $D$. If $\phi: D \rightarrow R$ is a real function such that

$$
\varphi[t x+(1-t) y]>t \varphi(x)+(1-t) \varphi(y), \quad \forall x, y \in D, x \neq y, t \in(0,1),
$$

then $\phi$ is called strictly concave function on $D$.

\section{Main results}

We are now in the position to apply the topological degree and properties of strictly convex (concave) function to derive some new fixed point theorems for semi-closed 1set-contractive operators and existence theorems of solutions for the equation $A x=\mu x$ which generalize a great deal of well-known results and relevant recent ones.

Theorem 2.1. Let $E, \Omega, A$ be the same as in Lemma 1.1. Moreover, if there exist strictly convex function $\phi: R^{+} \rightarrow R^{+}$with $\phi(0)=0$ and real function $\varphi: R^{+} \rightarrow R$ with $\varphi(t) \geq 1$, for all $t>1$, such that

$$
\varphi(\|A x-x\|) \geq \varphi(\| A x||) \phi\left(\|A x\| \cdot\|x\|^{-1}\right)-\varphi(\|x\|), \quad \forall x \in \partial \Omega,
$$

then $\operatorname{deg}(I-A, \Omega, \theta)=1$ if $A$ has no fixed point on $\partial \Omega$, and so $A$ has at least one fixed point in $\bar{\Omega}$.

Proof. If the operator $A$ has a fixed point on $\partial \Omega$, then $A$ has at least one fixed point in $\bar{\Omega}$. Now suppose that $A$ has no fixed points on $\partial \Omega$. Next we shall prove that the condition (L-S) is satisfied.

Suppose this is not true. Then there exists $x_{0} \in \partial \Omega, t_{0} \geq 1$ such that $A x_{0}=t_{0} x_{0}$, i.e., $x_{0}=t_{0}^{-1} A x_{0}$. It is easy to see that $\left\|A x_{0}\right\| \neq 0$ and $t_{0}>1$.

From (1), we have

$$
\varphi\left(\left\|A x_{0}-t_{0}^{-1} A x_{0}\right\|\right) \geq \varphi\left(\left\|A x_{0}\right\|\right) \phi\left(\left\|A x_{0}\right\| \cdot\left\|t_{0}^{-1} A x_{0}\right\|^{-1}\right)-\varphi\left(\left\|t_{0}^{-1} A x_{0}\right\|\right),
$$

which implies

$$
\varphi\left[\left(1-t_{0}^{-1}\right)\left\|A x_{0}\right\|\right]+\varphi\left(t_{0}^{-1}\left\|A x_{0}\right\|\right) \geq \varphi\left(\left\|A x_{0}\right\|\right) \phi\left(t_{0}\right) .
$$

By strict convexity of $\phi$ and $\phi(0)=0$, we obtain

$$
\begin{aligned}
\varphi\left[\left(1-t_{0}^{-1}\right)\left\|A x_{0}\right\|\right]+\varphi\left(t_{0}^{-1}\left\|A x_{0}\right\|\right) & =\varphi\left[\left(1-t_{0}^{-1}\right)\left\|A x_{0}\right\|+t_{0}^{-1}\|\theta\|\right]+\varphi\left[t_{0}^{-1}\left\|A x_{0}\right\|+\left(1-t_{0}^{-1}\right)\|\theta\|\right] \\
& <\left(1-t_{0}^{-1}\right) \varphi\left(\left\|A x_{0}\right\|\right)+t_{0}^{-1} \varphi(0)+t_{0}^{-1} \varphi\left(\left\|A x_{0}\right\|\right)+\left(1-t_{0}^{-1}\right) \varphi(0) \\
& =\varphi\left(\left\|A x_{0}\right\|\right) .
\end{aligned}
$$

It is easy to see from (2) and (3) that

$$
\varphi\left(\left\|A x_{0}\right\|\right) \phi\left(t_{0}\right)<\varphi\left(\left\|A x_{0}\right\|\right) .
$$

Noting that $t_{0}>1$ and $\varphi(t) \geq 1$, for all $t>1$, we have

$$
\varphi\left(\left\|A x_{0}\right\|\right) \phi\left(t_{0}\right) \geq \varphi\left(\left\|A x_{0}\right\|\right),
$$

which contradicts (4), and so the condition (L-S) is satisfied. Therefore, it follows from Lemma 1.1 that the conclusions of Theorem 2.1 hold. $\square$

Remark 2.2. If there exist convex function $\phi: R^{+} \rightarrow R^{+}, \phi(0)=0$ and real function $\varphi$ $: R^{+} \rightarrow R, \varphi(t)>1, \forall t>1$ satisfied (1), the conclusions of Theorem 2.1 also hold.

Theorem 2.3. Let $E, \Omega, A$ be the same as in Lemma 1.1. Moreover, if there exist strictly concave function $\phi: R^{+} \rightarrow R^{+}$with $\phi(0)=0$ and real function $\varphi: R^{+} \rightarrow R, \varphi$ $(t) \leq 1, \forall t>1$, such that 


$$
\varphi(\|A x-x\|) \leq \varphi(\|A x\|) \phi\left(\|A x\| \cdot\|x\|^{-1}\right)-\varphi(\|x\|), \quad \forall x \in \partial \Omega,
$$

then $\operatorname{deg}(I-A, \Omega, \theta)=1$ if $A$ has no fixed point on $\partial \Omega$, and so $A$ has at least one fixed point in $\bar{\Omega}$.

Proof. If the operator $A$ has a fixed point on $\partial \Omega$, then $A$ has at least one fixed point in $\bar{\Omega}$. Now suppose that $A$ has no fixed points on $\partial \Omega$. Next we shall prove that the condition (L-S) is satisfied.

Suppose this is not true. Then there exists $x_{0} \in \partial \Omega, t_{0} \geq 1$ such that $A x_{0}=t_{0} x_{0}$, i.e., $x_{0}=t_{0}^{-1} A x_{0}$. It is easy to see that $\left\|A x_{0}\right\| \neq 0$ and $t_{0}>1$. From (5), we have

$$
\varphi\left(\left\|A x_{0}-t_{0}^{-1} A x_{0}\right\|\right) \leq \varphi\left(\left\|A x_{0}\right\|\right) \phi\left(\left\|A x_{0}\right\| \cdot\left\|t_{0}^{-1} A x_{0}\right\|^{-1}\right)-\varphi\left(\left\|t_{0}^{-1} A x_{0}\right\|\right) .
$$

This implies that

$$
\varphi\left[\left(1-t_{0}^{-1}\right)\left\|A x_{0}\right\|\right]+\varphi\left(t_{0}^{-1}\left\|A x_{0}\right\|\right) \leq \varphi\left(\left\|A x_{0}\right\|\right) \phi\left(t_{0}\right) .
$$

By strict concavity of $\phi$ and $\phi(0)=0$, we obtain

$$
\begin{aligned}
\varphi\left[\left(1-t_{0}^{-1}\right)\left\|A x_{0}\right\|\right]+\varphi\left(t_{0}^{-1}\left\|A x_{0}\right\|\right) & =\varphi\left[\left(1-t_{0}^{-1}\right)\left\|A x_{0}\right\|+t_{0}^{-1}\|\theta\|\right]+\varphi\left[t_{0}^{-1}\left\|A x_{0}\right\|+\left(1-t_{0}^{-1}\right)\|\theta\|\right] \\
& >\left(1-t_{0}^{-1}\right) \varphi\left(\left\|A x_{0}\right\|\right)+t_{0}^{-1} \varphi(0)+t_{0}^{-1} \varphi\left(\left\|A x_{0}\right\|\right)+\left(1-t_{0}^{-1}\right) \varphi(0) \\
& =\varphi\left(\left\|A x_{0}\right\|\right) .
\end{aligned}
$$

It follows from (6) and (7) that

$$
\varphi\left(\left\|A x_{0}\right\|\right) \phi\left(t_{0}\right)>\varphi\left(\left\|A x_{0}\right\|\right) .
$$

On the other hand, by $t_{0}>1$ and $\varphi(t) \leq 1, \forall t>1$, we have

$$
\varphi\left(\left\|A x_{0}\right\|\right) \phi\left(t_{0}\right) \leq \varphi\left(\left\|A x_{0}\right\|\right)
$$

which contradicts (8), and so the condition (L-S) is satisfied. Therefore, it follows from Lemma 1.1 that the conclusions of Theorem 2.3 hold. $\square$

Remark 2.4. If there exist concave function $\phi: R^{+} \rightarrow R^{+}, \phi(0)=0$ and real function $\varphi: R^{+} \rightarrow R, \varphi(t)<1, \forall t>1$ satisfied (5), the conclusions of Theorem 2.3 also hold.

Corollary 2.5. Let $E, \Omega, A$ be the same as in Lemma 1.1. Moreover, if there exist $\alpha \in$ $(-\infty, 0) \cup(1,+\infty)$ and $\beta \geq 0$ such that

$$
\|A x-x\|^{\alpha} \geq\|A x\|^{\alpha+\beta}\|x\|^{-\beta}-\|x\|^{\alpha}, \quad \forall x \in \partial \Omega,
$$

then $\operatorname{deg}(I-A, \Omega, \theta)=1$ if $A$ has no fixed point on $\partial \Omega$, and so $A$ has at least one fixed point in $\bar{\Omega}$.

Proof. Putting $\phi(t)=t^{\alpha}, \varphi(t)=t^{\beta}$, we have $\phi(t)$ is a strictly convex function with $\phi$ $(0)=0$ and $\varphi(t) \geq 1, \forall t>1$. Therefore, from Theorem 2.1, the conclusions of Corollary 2.5 hold.. $\square$

Remark 2.6. 1. Corollary 2.5 generalizes Theorem 2.2 of $\mathrm{Xu}$ [7] from $\alpha>1$ to $\alpha \in$ $(-\infty, 0) \cup(1,+\infty)$. Moreover, our methods are different from those in many recent works (e.g., $\mathrm{Li}[6], \mathrm{Xu}[7])$.

2. Putting $\alpha>1, \beta=0$ in Corollary 2.5, we can obtain Theorem 5 of Li [6].

Corollary 2.7. Let E, $\Omega, A$ be the same as in Lemma 1.1. Moreover, if there exist $\alpha \in$ $(0,1)$ and $\beta \leq 0$ such that

$$
\|A x-x\|^{\alpha} \leq\|A x\|^{\alpha+\beta}\|x\|^{-\beta}-\|x\|^{\alpha}, \quad \forall x \in \partial D,
$$


then $\operatorname{deg}(I-A, \Omega, \theta)=1$ if $A$ has no fixed point on $\partial \Omega$, and so $A$ has at least one fixed point in $\bar{\Omega}$.

Proof. Putting $\phi(t)=t^{\alpha}, \varphi(t)=t^{\beta}$, we have $\phi(t)$ is a strictly concave function with $\phi$ $(0)=0$ and $\varphi(t) \leq 1, \forall t>1$. Therefore, from Theorem 2.3, the conclusions of Corollary 2.7 hold. $\square$

Remark 2.8. Corollary 2.7 extends Theorem 8 of Li [6]. Putting $\beta=0$ in Corollary 2.7, we can obtain Theorem 8 of Li [6].

Theorem 2.9. Let $E, \Omega, A$ be the same as in Lemma 1.1. Moreover, if there exist strictly convex function $\phi: R^{+} \rightarrow R^{+}$with $\phi(0)=0$ and real function $\varphi: R^{+} \rightarrow R$ with $\varphi(t) \geq 1$, for all $t>1$, such that

$$
\varphi(\|A x-x\|) \geq \varphi(\|A x\|) \phi\left(\|A x+x\| \cdot\|x\|^{-1}\right)-\varphi(\|x\|), \quad \forall x \in \partial \Omega,
$$

then $\operatorname{deg}(I-A, \Omega, \theta)=1$ if $A$ has no fixed point on $\partial \Omega$, and so $A$ has at least one fixed point in $\bar{\Omega}$.

Proof. If the operator $A$ has a fixed point on $\partial \Omega$, then $A$ has at least one fixed point in $\bar{\Omega}$. Now suppose that $A$ has no fixed points on $\partial \Omega$. Next we shall prove that the condition (L-S) is satisfied.

Suppose this is not true. Then there exists $x_{0} \in \partial \Omega, t_{0} \geq 1$ such that $A x_{0}=t_{0} x_{0}$, i.e., $x_{0}=t_{0}^{-1} A x_{0}$. It is easy to see that $\left\|A x_{0}\right\| \neq 0$ and $t_{0}>1$. By virtue of (9), we have

$$
\varphi\left(\left\|A x_{0}-t_{0}^{-1} A x_{0}\right\|\right) \geq \varphi\left(\left\|A x_{0}\right\|\right) \phi\left(\left\|A x_{0}+t_{0}^{-1} A x_{0}\right\| \cdot\left\|t_{0}^{-1} A x_{0}\right\|^{-1}\right)-\varphi\left(\left\|t_{0}^{-1} A x_{0}\right\|\right),
$$

which implies

$$
\varphi\left[\left(1-t_{0}^{-1}\right)\left\|A x_{0}\right\|\right]+\varphi\left(t_{0}^{-1}\left\|A x_{0}\right\|\right) \geq \varphi\left(\left\|A x_{0}\right\|\right) \phi\left[\left(1+t_{0}^{-1}\right) t_{0}\right] .
$$

By strict convexity of $\phi$ and $\phi(0)=0$, we obtain (3) holds. From (3) and (10), we have

$$
\varphi\left(\left\|A x_{0}\right\|\right) \phi\left[\left(1+t_{0}^{-1}\right) t_{0}\right]<\varphi\left(\left\|A x_{0}\right\|\right) .
$$

Noting that $t_{0}>1$ and $\varphi(t) \geq 1$, for all $t>1$, we have $\left(1+t_{0}^{-1}\right) t_{0}=t_{0}+1>1$, and so

$$
\varphi\left(\left\|A x_{0}\right\|\right) \phi\left[\left(1+t_{0}^{-1}\right) t_{0}\right] \geq \varphi\left(\left\|A x_{0}\right\|\right),
$$

which contradicts (11), and so the condition (L-S) is satisfied. Therefore, it follows from Lemma 1.1 that the conclusions of Theorem 2.9 hold. $\square$

Remark 2.10. If there exist convex function $\phi: R^{+} \rightarrow R^{+}, \phi(0)=0$ and real function $\varphi: R^{+} \rightarrow R, \varphi(t)>1, \forall t>1$ satisfied (9), the conclusions of Theorem 2.9 also hold.

Theorem 2.11. Let $E, \Omega, A$ be the same as in Lemma 1.1. Moreover, if there exist strictly concave function $\phi: R^{+} \rightarrow R^{+}$with $\phi(0)=0$ and real function $\varphi: R^{+} \rightarrow R, \varphi$ $(t) \leq 1, \forall t>1$, such that

$$
\varphi(\|A x-x\|) \leq \varphi(\|A x\|) \phi\left(\|A x+x\| \cdot\|x\|^{-1}\right)-\varphi(\|x\|), \quad \forall x \in \partial \Omega,
$$

then $\operatorname{deg}(I-A, \Omega, \theta)=1$ if $A$ has no fixed point on $\partial \Omega$, and so $A$ has at least one fixed point in $\bar{\Omega}$.

Proof. If the operator $A$ has a fixed point on $\partial \Omega$, then $A$ has at least one fixed point in $\bar{\Omega}$. Now suppose that $A$ has no fixed points on $\partial \Omega$. Next we shall prove that the condition (L-S) is satisfied. 
Suppose this is not true. Then there exists $x_{0} \in \partial \Omega, t_{0} \geq 1$ such that $A x_{0}=t_{0} x_{0}$, i.e., $x_{0}=t_{0}^{-1} A x_{0}$. It is easy to see that $\left\|A x_{0}\right\| \neq 0$ and $t_{0}>1$. By (12), we have

$$
\varphi\left(\left\|A x_{0}-t_{0}^{-1} A x_{0}\right\|\right) \leq \varphi\left(\left\|A x_{0}\right\|\right) \phi\left(\left\|A x_{0}+t_{0}^{-1} A x_{0}\right\| \cdot\left\|t_{0}^{-1} A x_{0}\right\|^{-1}\right)-\varphi\left(\left\|t_{0}^{-1} A x_{0}\right\|\right),
$$

which implies

$$
\varphi\left[\left(1-t_{0}^{-1}\right)\left\|A x_{0}\right\|\right]+\varphi\left(t_{0}^{-1}\left\|A x_{0}\right\|\right) \leq \varphi\left(\left\|A x_{0}\right\|\right) \phi\left[\left(1+t_{0}^{-1}\right) t_{0}\right] .
$$

By strict concavity of $\phi$ and $\phi(0)=0$, we have (7) holds. From (7) and (13), we obtain

$$
\varphi\left(\left\|A x_{0}\right\|\right) \phi\left[\left(1+t_{0}^{-1}\right) t_{0}\right]>\varphi\left(\left\|A x_{0}\right\|\right) .
$$

On the other hand, by $t_{0}>1$, we have $\left(1+t_{0}^{-1}\right) t_{0}=t_{0}+1>1$. Therefore, it follows from $\varphi(t) \leq 1, \forall t>1$ that

$$
\varphi\left(\left\|A x_{0}\right\|\right) \phi\left[\left(1+t_{0}^{-1}\right) t_{0}\right] \leq \varphi\left(\left\|A x_{0}\right\|\right),
$$

which contradicts (14), and so the condition (L-S) is satisfied. Therefore, it follows from Lemma 1.1 that the conclusions of Theorem 2.11 hold.

Remark 2.12. If there exist convex function $\phi: R^{+} \rightarrow R^{+}, \phi(0)=0$ and real function $\varphi: R^{+} \rightarrow R, \varphi(t)>1, \forall t>1$ satisfied (12), the conclusions of Theorem 2.11 also hold.

Corollary 2.13. Let $E, \Omega, A$ be the same as in Lemma 1.1. Moreover, if there exist $\alpha$ $\in(-\infty, 0) \cup(1,+\infty)$ and $\beta \geq 0$ such that

$$
\|A x-x\|^{\alpha}|| x||^{\beta} \geq\|A x\|^{\alpha}\|A x+x\|^{\beta}-\|x\|^{\alpha+\beta}, \quad \forall x \in \partial \Omega,
$$

then $\operatorname{deg}(I-A, \Omega, \theta)=1$ if $A$ has no fixed point on $\partial \Omega$, and so $A$ has at least one fixed point in $\bar{\Omega}$.

Proof. From (15), we have

$$
\left\|A x-x||^{\alpha} \geq\right\| A x\left\|^{\alpha}|| A x+x||^{\beta}|| x||^{-\beta}-\right\| x \|^{\alpha}, \quad \forall x \in \partial \Omega .
$$

Taking $\phi(t)=t^{\alpha}, \varphi(t)=t^{\beta}$, we have $\phi(t)$ is a strictly convex function with $\phi(0)=0$ and $\varphi(t) \geq 1, \forall t>1$. Therefore, from Theorem 2.9, the conclusions of Corollary 2.13 hold. $\square$

Remark 2.14. 1. Corollary 2.13 generalizes Theorem 2.4 of Xu [7] from $\alpha>1$ to $\alpha \in$ $(-\infty, 0) \cup(1,+\infty)$. Moreover, our methods are different from those in many recent works (e.g., Li [6], Xu [7]).

2. Putting $\alpha>1, \beta=0$ in Corollary 2.13, we can obtain Theorem 5 of Li [6].

Corollary 2.15. Let E, $\Omega, A$ be the same as in Lemma 1.1. Moreover, if there exist $\alpha$ $\in(0,1)$ and $\beta \leq 0$ such that

$$
\|A x-x\|^{\alpha}|| x||^{\beta} \leq\|A x\|^{\alpha}\|A x+x\|^{\beta}-\|x\|^{\alpha+\beta}, \quad \forall x \in \partial \Omega,
$$

then $\operatorname{deg}(I-A, \Omega, \theta)=1$ if $A$ has no fixed point on $\partial \Omega$, and so $A$ has at least one fixed point in $\bar{\Omega}$.

Proof. From (16), we have

$$
\|A x-x\|^{\alpha} \leq\left\|A x||^{\alpha}\right\| A x+x\left\|^{\beta}|| x\right\|^{-\beta}-\|x\|^{\alpha}, \quad \forall x \in \partial \Omega .
$$

Putting $\phi(t)=t^{\alpha}, \varphi(t)=t^{\beta}$, we have $\phi(t)$ is a strictly concave function with $\phi(0)=0$ and $\varphi(t) \leq 1, \forall t>1$. Therefore, from Theorem 2.11, the conclusions of Corollary 2.15 hold. $\square$ 
Remark 2.16. Corollary 2.15 extends Theorem 8 of Li [6]. Putting $\beta=0$ in Corollary 2.15, we can obtain Theorem 8 of Li [6].

Theorem 2.17. Let $E, \Omega, A$ be the same as in Lemma 1.1. Moreover, if there exist $\alpha$ $\in(-\infty, 0) \cup(1,+\infty), \beta \geq 0$ and $\mu \geq 1$ such that

$$
\|A x-\mu x\|^{\alpha} \geq\|A x\|^{\alpha+\beta}\|\mu x\|^{-\beta}-\|\mu x\|^{\alpha}, \quad \forall x \in \partial \Omega,
$$

then the equation $A x=\mu x$ possesses a solution in $\bar{\Omega}$.

Proof. Without loss of generality, suppose that $\frac{1}{\mu} A$ has no fixed point on $\partial \Omega$. From (17), we have

$$
\frac{1}{\mu^{\alpha}}\|A x-\mu x\|^{\alpha} \geq \frac{1}{\mu^{\alpha}}\|A x\|^{\alpha+\beta}\|\mu x\|^{-\beta}-\frac{1}{\mu^{\alpha}}\|\mu x\|^{\alpha}, \quad \forall x \in \partial \Omega,
$$

which implies

$$
\left\|\frac{1}{\mu} A x-x\right\|^{\alpha} \geq\left\|\frac{1}{\mu} A x\right\|^{\alpha+\beta}\|x\|^{-\beta}-\|x\|^{\alpha}, \quad \forall x \in \partial \Omega .
$$

It is easy to see that $\frac{1}{\mu} A$ is a semi-closed 1 -set-contractive operator. It follows from Corollary 2.5 that $\operatorname{deg}\left(I-\frac{1}{\mu} A, \Omega, \theta\right)=1 \neq 0$, and so the equation $A x=\mu x$ possesses a solution in $\bar{\Omega}$.

Remark 2.18. Similarly, from Corollary 2.7, Corollary 2.13 or Corollary 2.15, we can obtain the equation $A x=\mu x$ possesses a solution in $\bar{\Omega}$.

\section{Acknowledgements}

This study was supported by the Natural Science Foundation of Yancheng Teachers University under Grant (10YCKL022).

\section{Competing interests}

The authors declare that they have no competing interests.

Received: 14 November 2010 Accepted: 19 July 2011 Published: 19 July 2011

\section{References}

1. Petryshyn, W: Remark on condensing and k-set-contractive mappings. J. Math. Anal. Appl. 39, 717-741 (1972). doi:10.1016/0022-247X(72)90194-1

2. Li, GZ: The fixed point index and the fixed point theorems for 1- set-contraction mappings. Proc. Am. Math. Soc. 104 1163-1170 (1988)

3. Nussbaum, RD: Degree theory for local condensing maps. J. Math. Anal. Appl. 37, 741-766 (1972). doi:10.1016/0022247X(72)90253-3

4. Amann, H: Fixed point equations and nonlinear eigenvalue problems in ordered Banach space. SIAM Rev. 18, 620-709 (1976). doi:10.1137/1018114

5. Nussbaum, RD: The fixed index and asymptotic fixed point theorems for k-set-contractions. Bull. Am. Math. Soc. 75 490-495 (1969). doi:10.1090/50002-9904-1969-12213-5

6. Li, GZ, Xu, SY, Duan, HG: Fixed point theorems for 1-set-contractive operators in Banach spaces. Appl. Math. Lett. 19, 403-412 (2006). doi:10.1016/j.aml.2005.02.035

7. Xu, SY: New fixed point theorems for 1-set-contractive operators in Banach spaces. Nonlinear Anal. 67, $938-944$ (2007). doi:10.1016/j.na.2006.06.051

doi:10.1186/1687-1812-2011-15

Cite this article as: Wang: The fixed point theorems of 1-set-contractive operators in Banach space. Fixed Point Theory and Applications 2011 2011:15. 